Proc. XII Int. School on Theoretical Physics — Symmetry and Structural Properties of Condensed Matter

\title{
Disentanglement and Black Holes: Information Problem
}

\author{
A. RAdosz ${ }^{a, *}$, P. Gusin ${ }^{b}$ AND K. RoszaK ${ }^{c}$ \\ ${ }^{a}$ Department of Quantum Technologies, Wrocław University of Science and Technology, Wrocław, Poland \\ ${ }^{b}$ Faculty of Technology and Computer Science, Wrocław University of Science and Technology, Wrocław, Poland \\ ${ }^{c}$ Department of Theoretical Physics, Wrocław University of Science and Technology, Wrocław, Poland
}

One of the possible ways of formulation of an information loss paradox refers to an entanglement of the two particles created in a vicinity of an event horizon. Evolution of the entangled particles and an interaction with their own environments should lead to a decay of the entanglement. However obvious, such a perspective appears to be too naive in this case.

DOI: 10.12693/APhysPolA.132.132

PACS/topics: 04.70.Dy, 03.65.Ud

\section{Introduction}

Information problem has become for more than forty years and still is an unresolved paradox. It was indicated by Hawking [1] that apart of the well-known uncertainty principle in quantum mechanics there exists another source of uncertainty in physics, non-quantum origin: that is a black hole, with its event horizon regarded as a semitransparent "membrane". When the matter or radiation infalling towards a black hole, crosses its horizon the information it carries is lost. This led to the two kinds of problems. First was the controversial thesis: the information itself is assumed to be lost. Second, appeared to be even more striking controversy associated with fundamentals of quantum theory. Indeed, one can assume formation of a black hole due to a gravitational collapse of a matter (or radiation) is a unitary evolution of a pure quantum mechanical state. However, after formation of the event horizon, further evolution has to be regarded in the terms of mixed but not pure states, due to the loss of information; this means the breakdown of the quantum mechanical predictability.

Both of these outcomes of the Hawking idea had been later objected. The interesting contribution was another Hawking discovery: quantum mechanical effect related to the property of the event horizon. The fact that one of the two Killing vectors changes its properties, it is timelike outside event horizon and space-like inside horizon, opens the possibility of the creation of pairs of particles in vicinity of a horizon. One of these two falls in beyond the horizon and the other goes away to the infinity where it may be recorded - it is the so-called Hawking radiation $[2,3]$. The Hawking radiation was found to be a blackbody radiation of the temperature proportional to the inverse mass of a black hole.

The consequence of the energy conservation in this case: the release of the radiation leads to reduction of the mass of a black hole, was the entropy production. The other one was underlined by Page, who indicated [4]

*corresponding author; e-mail: andrzej.radosz@pwr.edu.pl that formally, evolution of a black hole meant as formation and evaporation, should obey quantum mechanical predictability. The following microscopic approach seemed to uncover no information retrieved due to the Hawking radiation. This result was then critically reviewed by Page $[4,5]$. It was shown that at the initial stages of a black hole evaporation both radiation as well as the black hole were at their nearly maximally mixed states: radiation does not carry the information. The process of releasing the information turned out to be of non-perturbative character. These kinds of arguments led to the formulation of the idea of black hole complementarity (BHC).

Recently there was formulated a hypothesis objecting BHC. It was shown that the claim of the quantum mechanical unitary evolution of the black hole may result in the breakdown of the smoothness of an event horizon neighborhood, leading to a "firewall" [6]. The firewall concept itself has been critically considered from different point of views in more recent studies [7-11].

There are variety of possible formulations of the information problem. In this paper (see also [12]) we will discuss one of them. Namely, during evaporation of a black hole pairs of entangled particles are created. Further, the one of positive energy tunnels through the horizon and the other one, of negative energy falls in and reduces mass of the black hole. Both of these entangled particles are evolving within their own environments. This should result in partial or complete disentanglement.

\section{Entropy, evaporation, information}

As indicated by Bekenstein and then shown by Hawking entropy of a black hole is proportional to its surface area, $A$ expressed in the Planck units

$$
S_{\mathrm{BH}}=k_{\mathrm{B}} \frac{A}{4 l_{\mathrm{Pl}}^{2}},
$$

where $A=4 \pi R_{S}^{2}$ is the area of the sphere with radius $R_{S}=2 G M / c^{2}$ and $l_{\mathrm{Pl}}=\sqrt{G \hbar / c^{3}}$ is the Planck length.

One can regard black holes as thermodynamic systems and according to the so-called "no-hair" theorem they may be characterized by mass, angular momentum, and charge only. In this paper we will consider the case of 
the Schwarzschild spacetime where the only relevant parameter characterizing black hole is its mass $M$. Then applying thermodynamic relation, $\mathrm{d} U=T \mathrm{~d} S$, and using the fact that $U=M$, one obtains here that the temperature of the black hole of mass $M$ is

$$
T_{\mathrm{BH}}=\hbar c^{3} /\left(8 \pi k_{\mathrm{B}} G M\right) \text {. }
$$

As pointed out by Hawking [2] in the vicinity of the horizon, pair of the particles might be created and annihilated but if one member of a pair goes to infinity, the other member of the pair falls in. Such a pair must contain a positive energy member, the former one, and a negative energy member, the one that penetrates the black hole interior. It was explained later [13] that characteristic feature of these phenomena is a tunnelling through the barrier: if the particles are created outside horizon, the negative energy particles tunnels and falls in and when they arise inside horizon then the positive energy one tunnels through and goes away. The radiation recorded by distant observer, the Hawking radiation turns out to be blackbody radiation of the temperature $T_{\mathrm{BH}}$ (see above). This process results in a reduction of mass of the black hole - evaporation. It is assumed that the black hole radiates according to the Stefan-Boltzmann law

$$
\frac{\mathrm{d} P}{\mathrm{~d} A}=\sigma T_{\mathrm{BH}}^{4},
$$

where $\sigma=\pi^{2} k_{\mathrm{B}}^{4} /\left(60 \hbar^{3} c^{2}\right)$. Hence, one can describe evaporation as follows:

$$
\frac{\mathrm{d} M}{\mathrm{~d} t}=-\frac{\varepsilon}{3} \frac{1}{M^{2}}
$$

with the solution $M^{3}(t)=M_{0}^{3}-\varepsilon t$, where $M_{0}$ is the initial mass of the black hole and $\varepsilon=\hbar c^{4} /\left(2^{10} \cdot 5 \pi G^{2}\right)=$ $m_{\mathrm{Pl}}^{3} /\left(2^{10} \cdot 5 \pi t_{\mathrm{Pl}}\right)$. Here we introduced the Planck time $t_{\mathrm{Pl}}=\sqrt{G \hbar / c^{5}}$ and the Planck mass $m_{\mathrm{Pl}}=\sqrt{\hbar c / G}$. The lifetime $\tau$ of black hole of mass $M_{0}$ is $\tau=M_{0}^{3} / \varepsilon$. During the evaporation process entropy of a black hole decreases: $\mathrm{d} S_{\mathrm{BH}}=-\mathrm{d} M / T_{\mathrm{BH}}$. But the overall entropy increases $[14,15]$, as the amount of the entropy of the radiation is by a third part greater: $\mathrm{d} S=4 \mathrm{~d} M /\left(3 T_{\mathrm{BH}}\right)$. The entropy $S_{\mathrm{BH}}$ as the function of time is

$$
S_{\mathrm{BH}}(t)=\frac{\pi k_{\mathrm{B}}}{16}\left(\frac{\tau-t}{10 \pi t_{\mathrm{Pl}}}\right)^{2 / 3} .
$$

It was argued by Page [4] that formation and evaporation of a black hole might be regarded as a unitary process and the information lost due to the presence of an event horizon is eventually retrieved. However, outflow of information from a black hole is nonperturbative, very slow process. Indeed, initially a leakage of information $I$ occurs in the following rate:

$$
\frac{\mathrm{d} I}{\mathrm{~d} t} \sim \exp \left(-S_{\mathrm{BH}}(t) / k_{\mathrm{B}}\right) .
$$

However using (2.3) we can express the last formula as the function of mass $M$ :

$$
\frac{\mathrm{d} I}{\mathrm{~d} M} \sim-\frac{3}{\varepsilon} M^{2} \exp \left(-4 \pi \frac{M^{2}}{m_{\mathrm{Pl}}^{2}}\right),
$$

where $M$ decreases from $M_{0}$ to 0 . Thus the information $I$ for the black hole with the mass $m<M_{0}$ is given by the integral

$$
\begin{gathered}
I(m) \sim \frac{3}{\varepsilon} \int_{m}^{M_{0}} M^{2} \exp \left(-4 \pi \frac{M^{2}}{m_{\mathrm{Pl}}^{2}}\right) \mathrm{d} M= \\
\frac{3 M_{0}^{3}}{\varepsilon} \int_{m / M_{0}}^{1} x^{2} \exp \left(-\alpha^{2} x^{2}\right) \mathrm{d} x,
\end{gathered}
$$

where $\alpha^{2}=4 \pi M_{0}^{2} / m_{\mathrm{Pl}}^{2}$. The integral is equal to [12]:

$$
I(m) \sim 2^{7} \cdot 15 t_{\mathrm{Pl}}\left(F(m)-F\left(M_{0}\right)\right),
$$
where

$$
F(m) \equiv \frac{m}{M_{0}} \exp \left(-\frac{4 \pi m^{2}}{m_{\mathrm{Pl}}^{2}}\right)-\frac{1}{4} \operatorname{erf}\left(\frac{2 \sqrt{\pi} m}{m_{\mathrm{Pl}}}\right)
$$

and $\operatorname{erf}(x)$ is the error function.

\section{The Hawking radiation: entangled pairs of particles and their disentanglement}

The Hawking radiation consists of particles recorded away from an event horizon that are entangled with a negative energy particles living within horizon. Creation of more and more generations of entangled pairs leads to more particles recorded far from the horizon that are entangled with diminishing black hole. Eventually black hole vanishes; then what are those positive energy particles entangled with?

This is yet another formulation information loss paradox. Having in mind this particular aspect, we are attempting here in looking for resolution of the information problem in terms of a possible disentanglement process.

Let us consider an entangled pair of particles, not specifying a degree of freedom being entangled, assuming the two state character of the corresponding variable. Hence the subject of the discussion will be a pair of qubits in entangled state

$$
|\psi\rangle=a|0\rangle|0\rangle+b|0\rangle|1\rangle+c|1\rangle|0\rangle+d|1\rangle|1\rangle,
$$

where specific relation between $b, c$ and $d$ corresponds to the maximally entangled state. One can ask then how the event horizon separating these qubits affects their entanglement.

In order to discuss an entanglement evolution, we will consider two environments, $E_{I}(I-$ interior $)$ and $E_{E}(E$ - exterior) one for each of the two, I and E, respectively, qubits. Due to interaction with an environment, each qubit density matrix evolves affecting the state of the two qubits initially described by a pure state density matrix resulting from (3.1). Here we will discuss the following simplified case.

Let us discuss the case of the initial state (3.1) determined as $a=d=0$ and $b=c=1 / \sqrt{2}$, i.e. its density matrix given as

$$
\rho_{E_{I}}=\frac{1}{2}\left(\begin{array}{llll}
0 & 0 & 0 & 0 \\
0 & 1 & 1 & 0 \\
0 & 1 & 1 & 0 \\
0 & 0 & 0 & 0
\end{array}\right) .
$$

One can assume that $I$-qubit due to the interaction with its $E_{I}$ environment, undergoes phase decoherence, realized via a pair of Krauss operators [16]: 


$$
\rho_{1}=\left(\begin{array}{ll}
0 & 0 \\
0 & x
\end{array}\right)
$$

and

$$
\rho_{2}=\left(\begin{array}{cc}
1 & 0 \\
0 & \sqrt{1-x^{2}}
\end{array}\right),
$$

where $x$ is an evolution (temporal) parameter. Then the density matrix (3.2) evolves as follows:

$$
\rho_{E_{I}}(x)=\frac{1}{2}\left(\begin{array}{cccc}
0 & 0 & 0 & 0 \\
0 & 1 & x & 0 \\
0 & x^{*} & 1 & 0 \\
0 & 0 & 0 & 0
\end{array}\right) .
$$

One can guess that this kind of behaviour of the density matrix manifest in a sense entanglement decay. To study this one should invoke entanglement measure. A suitable one appears to be a concurrence [17], defined as follows. One takes the matrix

$$
\left(\sigma_{y} \otimes \sigma_{y}\right) \rho_{E_{I}}^{*}(x)\left(\sigma_{y} \otimes \sigma_{y}\right) \rho_{E_{I}}^{*}(x)
$$

and determines its eigenvalues, $\lambda_{a}$. In this case one finds the following eigenvalues:

$$
\lambda_{0,1}=(1 \pm|x|) \text { and } \lambda_{2,3}=0 .
$$

Concurrence $C$, the measure of the entanglement, is defined as the maximal value of the two:

$$
C=\max \left(0, \lambda_{0}-\sum_{a=1}^{3} \lambda_{a}\right) .
$$

In this case

$$
C=|x| \text {. }
$$

Therefore, in this case of the phase decoherence of the two entangled particles initially being in the one of the Bell states, Eq. (3.2), one finds that their entanglement decays according to (3.7), i.e. as defined in terms of the decoherence parameter.

\section{Conclusions}

The information loss paradox is strongly linked to the process of evaporation of black holes due to the Hawking radiation. In some sense it might be then viewed as entanglement of the particles recorded outside horizon with a diminishing and eventually vanishing interior of the black hole. There have been proposed variety of the model treatments of this problem invoking an idea of stretched horizon, virtual and real qubits existing in horizons vicinity and others (e.g. [18]) (the most recent proposal to remove this paradox, comes from an idea that the black holes have hair — see [19]). In this paper we have argued that both entangled particles evolve within their own environments. Such an evolution might mean "decoherence". We have considered then a simplified model of a phase decoherence of an initially maximally entangled (one of the Bell) state(s). This simple example reveals temporal decays of the entanglement: the concurrence $C$ may evolve with time from 1 to 0 (complete disentanglement) or to some nonzero limit (partial disentanglement).

However, even this oversimplified example cannot be regarded as a model of the process of gradual disentanglement of a pair of Hawking particles created in the vicinity of the horizon.

The point is that one member of such a pair must fall behind the horizon and other one stays in "our" part of the universe. But then the temporal relation between them is broken: we could not study the properties behind the horizon. The observer inside the horizon could, in principle, receive the signals from outer space and may study the temporal evolution of the concurrence. However, it is not that observer who finds the information loss paradox!

Though the qubit-environment coupling appears to be an obvious source of the entanglement decay, i.e. partial or complete disentanglement of two members of a pair of entangled particles, one finds that a naively simple approach could not be applied for the studies of disentanglement of a pair of Hawking particles. This is due to the existence of an event horizon in this case. Above presented set of the problems seems to be interesting enough to be studied in the future.

\section{References}

[1] S.W. Hawking, Nature 248, 30 (1974).

[2] S.W. Hawking, Commun. Math. Phys. 43, 199 (1975).

[3] S.W. Hawking, Phys. Rev. D 14, 2460 (1976).

[4] D.N. Page, Phys. Rev. Lett. 71, 3743 (1993).

[5] D.N. Page, J. Cosmol. Astropart. Phys. 2013, 028 (2013).

[6] A. Almheiri, D. Marolf, J. Polchinski, J. Sully, J. High Energy Physics 2013, 62 (2013).

[7] D.A. Lowe, L. Thorlacius, Phys. Rev. D 88, 044012 (2013).

[8] T. Banks, W. Fischler, arx1208.4757.

[9] J. Maldacena, L. Susskind, Progr. Phys. 61, 781 (2013).

[10] S. Mathur, arXiv:1506.04342.

[11] G. 't Hooft, arXiv:1511.04427.

[12] P. Gusin, A. Radosz, Entanglement and Information (Loss) Paradox, unpublished.

[13] M.K. Parikh, F. Wilczek, Phys. Rev. Lett. 85, 5042 (2000).

[14] W.H. Zurek, Phys. Rev. Lett. 49, 1683 (1982).

[15] D.N. Page, Phys. Rev. Lett. 50, 1013 (1983).

[16] M.A. Nielsen, I.L. Chuang, Quantum Computation and Quantum Information, Cambridge University Press, 2000.

[17] W.K. Wootters, Quant. Inform. Comput. 1, 27 (2001).

[18] E. Verlinde, H. Verlinde, arXiv:1211.6913.

[19] S.W. Hawking, M. Perry, A. Strominger, PRL 116, 231301 (2016). 